\title{
Modelación matemática para optimizar la estructura de los cursos en las plataformas e-learning
}

DOI: https://doi.org/10.33262/ap.v1i1.17

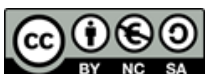

Mathematical modeling to optimize the structure of the courses in the platforms and-learning

Amaury Machín Armenteros., ${ }^{1}$ \& Yasser Vázquez Alfonso. ${ }^{2}$

\begin{abstract}
.
This investigation was carried out in the Agrarian University of the Fruitful Havana Rodríguez Pérez" (UNAH) located in the county of Mayabeque. He had as main objective to model the behavior of the users mathematically in the educational platforms for the optimization of the structure of the courses starting from the adjacency matrix and under the suppositions that at more time of permanency in a page of the same session, bigger interest in her, and that the frequency of use of the interrelations indicates a bigger utility of the same ones to consent toward the wanted information. Making use of Mining Web the necessary data was extracted to apply the pattern to the logs of the course Structure of Data II of the platform Moodle. The proposed pattern allows to optimize and to personalize the structure of the courses in the educational platforms; it is possible to find more efficient sailing structures from the point of the navigability and usability, improving the structure of the courses of the educational platforms in short in the UNAH.
\end{abstract}

Keywords: mathematical modeling, behavior, educational platforms, web mining, Moodle.

\footnotetext{
${ }^{1}$ Universidad de La Habana. La Habana, Cuba. Amaury Machín Armenteros

${ }^{2}$ Universidad de La Habana. La Habana, Cuba. Yasser Vázquez Alfonso
} 


\section{Resumen.}

Esta investigación se realizó en la Universidad Agraria de la Habana "Fructuoso Rodríguez Pérez" (UNAH) ubicada en la provincia de Mayabeque. Tuvo como principal objetivo modelar matemáticamente el comportamiento de los usuarios en las plataformas educativas para la optimización de la estructura de los cursos a partir de la matriz de adyacencia y bajo los supuestos que, a mayor tiempo de permanencia en una página de la misma sesión, mayor interés en ella, y que la frecuencia de uso de las interrelaciones indica una mayor utilidad de las mismas para acceder hacia la información deseada. Haciendo uso de Minería Web se extrajeron los datos necesarios para aplicar el modelo a los logs del curso Estructura de Datos II de la plataforma Moodle. El modelo propuesto permite optimizar y personalizar la estructura de los cursos en las plataformas educativas, se logra encontrar estructuras de navegación más eficientes desde el punto de la navegabilidad y usabilidad, mejorando en concreto la estructura de los cursos de las plataformas educativas en la UNAH.

Palabras claves: modelar matemáticamente, comportamiento, plataformas educativas, Minería web, Moodle.

\section{Introducción.}

El desarrollo de las nuevas tecnologías de la educación y la comunicación han hecho posible la utilización de Internet y más concretamente la WWW (World Wide Web) en la educación a distancia, dando lugar a la denominada Educación basada en Web o e-learning (Horton, 2000).

En el sentido literal e-learning significa aprendizaje electrónico, es decir, el aprendizaje producido a través de un medio tecnológico-digital. Rosenberg define elearning como el uso de las tecnologías basadas en Internet para proporcionar un amplio despliegue de soluciones que impactan en el aprendizaje y el rendimiento (Rosenberg, 2001 y Rosenberg, 2005). Otros autores como Clark y Mayer definen este concepto como la enseñanza transmitida desde un ordenador a través de los medios DVD-ROM, Internet, o intranet con las siguientes características (Clark and Mayer, 2011):

- Incluye contenido relevante para alcanzar los objetivos de aprendizaje.

- Utiliza métodos de enseñanza como ejemplos y ejercicios para ayudar en el aprendizaje.

- Utiliza elementos de comunicación como palabras e imágenes para transmitir el contenido y los métodos.

- Puede ser enseñanza dirigida por el profesor (e-learning síncrono) o diseñada para auto-aprendizaje (e-learning asíncrono).

- Sirve de ayuda a los estudiantes para conseguir sus objetivos educacionales. 
En los últimos años, e-learning supone una nueva concepción de la educación mediante la incorporación de nuevas tecnologías al proceso de enseñanza y aprendizaje. Esta plataforma educativa proporciona tanto a docentes como alumnos una importante variedad de recursos, lo que en muchas ocasiones esto suele ser una desventaja. Esto hace que el alumno se encuentre desorientando frente a la elección de recursos diseñados. Este problema requiere una reestructuración de cómo el docente ha diseñado la asignatura, con el objetivo de que ese diseño se adapte de mejor manera a las características e intereses de los alumnos (Corso and Alfaro, 2007).

El uso de ambientes virtuales basados en la web, como herramienta de apoyo al proceso educativo, se ha expandido en los últimos años, gracias al desarrollo de nuevos enfoques y modalidades de educación, como el e-learning y el b-learning. Estas nuevas formas de utilización de la tecnología como apoyo al proceso de enseñanza aprendizaje, han incentivado la aparición de software especializado en proveer los mecanismos que facilitan el desarrollo del trabajo autónomo del estudiante, su interacción con el docente, y el uso de herramientas colaborativas y de comunicación para el trabajo grupal (Aponte et al., 2012).

La introducción de estas tecnologías ha originado una proliferación de experiencias en los países de América Latina y el Caribe, que amerita un mayor y mejor conocimiento de sus características, con miras al mejoramiento de la calidad de la educación superior impartida a través de estas nuevas modalidades de trabajo académico (Machín;2016).

Para contribuir a satisfacer estas necesidades, a resolver esta problemática y mejorar la calidad de la educación superior en esta región a través de esas tecnologías, el Instituto Internacional de la Unesco para la Educación Superior en América Latina y el Caribe (IESALC) emprendió en el año 2002 un proyecto sobre la educación superior virtual y a distancia en esta región, el cual se insertó en su Programa Marco denominado Observatorio Sobre Educación Superior en América Latina y el Caribe (Silvio, 2004).

En Cuba, varias de sus universidades han creado diferentes centros de estudios para la investigación en TIC para la Educación Superior, tales como la Facultad de Educación a Distancia de la Universidad de La Habana , el Centro de Referencia en Educación Avanzada (CREA), donde se desarrolló un Modelo de Universidad Virtual CUJAE, la Facultad de Educación a Distancia de la Universidad Central de Las Villas, donde se desarrolló el Sistema de Enseñanza Personalizada a Distancia (SEPAD), el Centro de Estudio de Educación Superior Agraria (CEESA) de la Universidad Agraria de La Habana (Vázquez, 2008).

En la Universidad Agraria de La Habana "Fructuoso Rodríguez Pérez" (UNAH) desde hace varios años se viene trabajando en la aplicación de las TIC a la Educación Superior. Expresiones de esta es la creación de un Laboratorio de Tecnología Educativa (LATED), donde se desarrolla una amplia variedad de software educativos. Pero a pesar de los grandes 
esfuerzos realizados por crear un sistema que permita estudiar el comportamiento de los estudiantes mediante el cual el profesor pueda brindarle al estudiante lo que realmente necesita para potenciar sus conocimientos con el empleo de este tipo de tecnologías, aún no se cuenta con un sistema que permita analizar las preferencias y técnicas que usan los estudiantes para estudiar, lo cual posibilitaría perfeccionar la calidad educativa, ofreciendo a los alumnos un servicio más personalizado acorde con sus necesidades e intereses reales y de esta forma facilitar el aprendizaje mediante este tipo de plataformas en la universidad.

El quehacer que se desprende de la problemática antes planteada, hace que se plantee el siguiente objetivo general: Desarrollar un modelo matemático para la optimización de la estructura de los cursos en las plataformas e-learning de la UNAH.

\section{Metodología.}

En la investigación se utilizaron los métodos teóricos siguientes:

- Histórico lógico se realizó una reseña acerca de las características y utilización de los e-learning y los AG, así como de las principales técnicas utilizadas y aplicaciones respectivamente.

- Análisis y síntesis: consistió en un estudio pormenorizado de las características de los archivos log en los sistemas web, arribando a los elementos esenciales para su empleo en un modelo matemático que describa el comportamiento de los usuarios y optimice la estructura de los cursos.

- Enfoque de sistema se emplea para el estudio de los diferentes componentes de la Minería Web.

- Inducción-deducción: se emplea para la creación de un modelo matemático a partir del log permitiendo el análisis del comportamiento de los usuarios y la optimización de la estructura de los cursos en las plataformas e-learning.

Como método empírico se utilizará:

- Sondeo o levantamiento: se utilizó la técnica análisis documental para la recopilación de datos e información necesarios para el estudio del comportamiento de los usuarios y la optimización de la estructura de los cursos.

El método matemático que se utiliza es:

- Modelación matemática: se modeló a través de ecuaciones matemática utilizando el algoritmo genético para el estudio del comportamiento de los usuarios y la optimización de la estructura de los cursos en las plataformas e-learning de la UNAH.

\section{Datos web.}

Con el fin de anticiparse a la toma de decisiones, el análisis y entendimiento del comportamiento del usuario cobra una importancia fundamental, donde se hace necesario 
seguir en detalle sus acciones, las cuales podrían incluir toda la trayectoria de páginas visitadas, el tiempo gastado en cada una de ellas, los productos escogidos, cantidad de transiciones antes de llegar a concretar una compra, etc. (Velásquez and Palade, 2008). Así, con el hecho de navegar en la Web se van acumulando una serie de datos que proporcionarán información relevante: los registros web log (Ortega-Priego and Aguillo, 2009). Ellos contienen todo el recorrido de los usuarios, descargas asociadas (páginas, objetos multimedia, archivos, entre otros) y tiempo invertido. Estos se generan cuando se realiza la lectura del código HTML de cada página, que llega y se interpreta por el navegador, registrando cada objeto entregado. Es importante indicar que, aunque solo una página sea visitada, más de un objeto puede ser registrado (Velásquez and Palade, 2008), entre ellos una alta cantidad de objetos sin información relevante (banners publicitarios, íconos, etc.), aunque dicha relevancia dependerá del estudio que se quiera realizar, por lo que el filtrar y registrar previamente los datos de interés se vuelve una tarea clave.

En la Figura 2.1 se muestra un ejemplo de log de la plataforma Moodle en la UNAH.

Figura 1. Ejemplo de log de Moodle.

\begin{tabular}{|c|c|c|c|c|c|}
\hline Dirección IP & Nombre & Acción & \multicolumn{3}{|l|}{ Información } \\
\hline 2015 mayo 28 15:48 10.4.41.11 & Elio Minel del Pozo Nunez & course view & EVEA Agronomía & & \\
\hline 2015 mayo 28 15:45 10.4.41.11 & Elio Minel del Pozo Nunez & course view & Química General & & \\
\hline 2015 mayo 28 15:45 10.4.41.11 & Elio Minel del Pozo Nunez & course view & \multicolumn{2}{|c|}{ Química Inorgánica y Analítica } & \\
\hline 2015 mayo 28 15:44 10.4.41.11 & Elio Minel del Pozo Nunez & course view & \multicolumn{3}{|c|}{ Problemas Sociales de la Ciencia y la Tecnología } \\
\hline 2015 mayo 28 15:44 10.4.41.11 & Elio Minel del Pozo Nunez & course view & \multicolumn{2}{|c|}{ Economía Política del Capitalismo } & \\
\hline 2015 mayo $2815: 39$ 10.4.41.11 & Elio Minel del Pozo Nunez & course view & \multicolumn{2}{|c|}{ Ecología y Elementos de Agroecología } & \\
\hline 2015 mayo 11 10:29 10.4.132.143 & Arlenys Cruz Mendez & course view & \multicolumn{2}{|c|}{ Ecología y Elementos de Agroecología } & \\
\hline 2015 abril 23 4:20 10.4.40.4 & Arlenys Cruz Mendez & course view & \multicolumn{2}{|c|}{ Ecología y Elementos de Agroecología } & \\
\hline 2015 abril 23 4:20 10.4.40.4 & Arlenys Cruz Mendez & course view & \multicolumn{2}{|c|}{ Ecología y Elementos de Agroecología } & \\
\hline 2015 abril 8 7:27 10.4.15.6 & \multicolumn{2}{|c|}{ Alejandro Emilio Ramos Rodcourse view } & Proyecto agrícola & & \\
\hline 2015 abril $87: 26$ 10.4.15.6 & \multicolumn{2}{|c|}{ Alejandro Emilio Ramos Rod course view } & EVEA Agronomía & & \\
\hline 2015 abril $64: 07$ 10.4.40.2 & Hector Rodriguez Morell & course view & EVEA Agronomía & & \\
\hline 2015 abril $29: 01$ 10.4.128.121 & Valentin Garcia Castillo & course view & Práctica Agrícola I & & \\
\hline
\end{tabular}

Fuente: Elaboración propia.

\section{Proceso de sesionalización.}

Una sesión de usuario está definida por una secuencia de accesos temporales a un sitio particular de la web por el usuario (Menascé et al., 1999). Cada sesión de usuario es una representación lógica de una visita de un usuario a algún sitio web usualmente dentro algún intervalo de tiempo.

Entonces podemos definir una sesión de usuario $\mathrm{S}$ como el conjunto de páginas $\mathrm{P}$ visitadas:

$$
\mathrm{P}=\left\{\mathrm{p} 1, \mathrm{p} 2, \mathrm{p} 3, \ldots \ldots, \mathrm{p}_{\mathrm{n}}\right\}
$$


Teniendo los registros web log se hace necesario ahora estudiar las rutas de cada usuario individualizando sus sesiones. A aquel proceso de reconstrucción de caminos tomados se le llama sesionalización (Velásquez and Palade, 2008). El propósito del mismo, es encontrar las sesiones reales de cada usuario, por ello se han propuesto heurísticas para reconstruirlas desde el web log para asociar los registros a una sesión única durante un período de tiempo. Las técnicas ocupadas pueden ser clasificadas según la estrategia que siguen, ya sea proactiva o reactiva: Para la realización de este trabajo se utilizará una estrategia proactiva conocida como Cookies o simplemente la inscripción que realiza el usuario en el sitio web.

\section{Diseño del algoritmo.}

Si una compañía desea permanecer competitiva en el mercado digital, necesita un sitio web que ofrezca la información en concreto que los usuarios están buscando, de manera simple y accesible. Sin embargo, la contingencia indica que en muchos casos la estructura del sitio no ayuda a los usuarios a encontrar la información deseada, incluso cuando ésta si existe (Velásquez and Palade, 2008). Ante eso, y con el fin de determinar la mejor estructura de las plataformas e-learning, es que desde ahora se describe un modelo matemático que mediante el estudio del comportamiento de los usuarios (gracias a registros web log) permita determinar el mejor grafo para una navegación más eficiente (maximizando la utilidad de los enlaces existentes en base a los principios de usabilidad) en el sitio web.

Considerando los datos de entrada que se observan en la Tabla 1, cabe señalar preliminarmente los supuestos y observaciones propios de la extracción de datos y operación de los mismos.

Tabla 1: Datos de entrada del algoritmo.

\begin{tabular}{c}
\hline Datos \\
\hline Número total de páginas \\
Sesiones de usuario \\
Matriz de adyacencia = existencia de enlaces o links \\
Rutas de las páginas visitadas \\
Tiempo de permanencia en cada página \\
\hline
\end{tabular}

Fuente: Elaboración propia.

\section{Supuestos y Observaciones.}

1. A mayor tiempo de permanencia en una página de una misma sesión, mayor interés tiene el usuario en ella (Velásquez and Palade, 2008).

2. Sesiones de largo 1 no se consideran. 
3. No se considera la comparación de secuencias totales (clúster de sesiones (Velásquez and Palade, 2008)), es decir, se consideran las relaciones entre las de páginas en cuanto a frecuencias de uso.

4. No se considera la existencia de más de un enlace en una misma página i saliendo a otra j, se tomará como un solo camino presente entre dichas páginas.

5. Los cálculos son construidos en base a los registros de las visitas al sitio considerando cada transición realizada, es decir, se considera el comportamiento de los usuarios para la construcción de sus sesiones, teniendo implícito por lo tanto un cálculo basado en el uso.

\section{Parámetros del algoritmo.}

Los parámetros son aquellos valores de entrada que no cambian a lo largo de la ejecución del algoritmo y que dependen en gran medida de las etapas a implementar. En el caso de los AG se hace necesario definir:

\section{Tamaño de los individuos.}

En este caso, corresponde a un vector fila de largo mxm columnas, donde $\mathrm{m}$ es el número total de páginas del sitio. Sus valores binarios internos representan la existencia o no de enlaces entre páginas.

\section{Tamaño de la población.}

Indica cuántos individuos habrá en cada generación. Desde el punto de vista algorítmico, tiene que ver con el espacio de las soluciones posibles a estudiar.

\section{Criterio de detención.}

Son aquellos criterios por los cuales se establece que el algoritmo termine. Estos pueden ser fijando un número máximo de iteraciones que el algoritmo realice (número máximo de generaciones), indicando un tiempo límite de ejecución del mismo precisando un fitness límite (se detiene el algoritmo al encontrar valores menores o iguales a dicho fitness), detallando una función de tolerancia que considere el cambio acumulado en el valor del fitness a través de las generaciones, o bien, considerando intervalos de tiempo máximo. De esta manera, si no hay mejoras en la función objetivo, se detiene el algoritmo.

Los restantes parámetros corresponden a los operadores genéticos propios de los AG, los cuales dependerán del estudio que se desea realizar.

\section{Fitness scaling.}

Especificación de la escala a realizar entre todos los individuos de modo de compararlos en base a dicha escala. 


\section{Selección.}

Elección de los padres de la próxima generación, basado en su valor fitness escalado.

\section{Reproducción.}

Forma en que se crearán los hijos, especificando qué individuos tendrán sobrevivencia garantizada, qué porcentaje serán producidos vía cruce y qué porcentaje vía mutación.

\section{Mutación.}

Porcentaje de cambio aleatorio en los individuos de la población, el cual provee diversidad genética y explorar un espacio más amplio.

\section{Cruce.}

Forma de combinación de los padres, para formar un nuevo individuo.

\section{Resultados.}

Para la aplicación del modelo matemático se utilizó la información de los registros log del curso Estructura de Datos II (ver figura 2), perteneciente a la carrera de Ingeniería en informática.

Figura 2: Imagen del curso Estructura de Datos II.

\section{Tema II: El Tipo de Dato Abstracto Grafo}

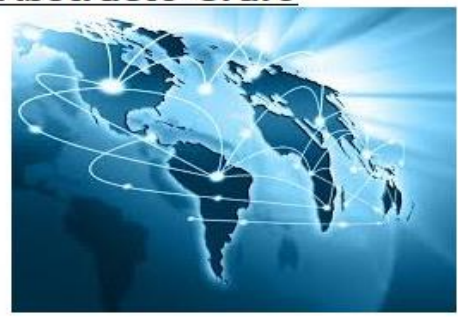

En matemáticas y ciencias de la computación, un grafo (del griego grafos: dibujo, imagen) es un conjunto de objetos llamados vértices o nodos unidos por enlaces llamados aristas o arcos, que permiten representar relaciones binarias entre elementos de un conjunto.

띨 10. Conferencia 3. Grafos. Definiciones y representación

댈 10. Conferencia 3. Presentación electrónica

삘 11. Clase Práctica 8 . Aplicación de las definiciones del TDA Grafo

[iv] 12. Clase práctica 9. Solución de problemas utilizando el TDA Grafo, con la representación por lista de adyacencia

뜰 13. Clase práctica 10. Aplicación de las definiciones del TDA utilizando la representación por matriz de adyacencia.

iㅣㄹ 14. Clase práctica 11. Solución de problemas utilizando el TDA Grafo, con la representación por matriz de adyacencia

띨] 15. Conferencia 4. Recorridos para grafos

댈 15. Conferencia 4. Recorridos para grafos

띨 16. Clase práctica 12. Implementación algoritmo BPA. Lista de adyacencia

Wiv] 17. Clase práctica 13. Implementación algoritmo BPA. Matriz de adyacencia

삘 18. Clase Practica 14. Implementacion BPP - Lista de adyacencia

Fuente: Plataforma Moodle. 

abril-junio, 2019

Este curso se encuentra publicado en la plataforma Moodle (Modular Object Oriented Developemental Learning Environment) en la Universidad Agraria de la Habana, la dirección principal del sitio es http://evea.unah.edu.cu/. Con el objetivo de aplicar el algoritmo se tomó una muestra de las 9 primeras páginas visitadas del curso, las cuales se codificaron de la siguiente manera:

1. Home

2. Árboles binarios

3. Conferencia 2. Árboles Binarios de Búsqueda

4. Conferencia 3. Grafos. Definiciones y representación

5. Conferencia 4. Recorridos para grafos

6. Materiales Complementarios/Recorridos para grafos

7. Recursividad

8. Data Structures and Algorithms

9. Clase práctica 5. Aplicación las definiciones del árbol binario de búsqueda.

Con el objetivo de realizar todo este proceso automáticamente, se utilizó un programa informático utilizando el lenguaje Java a través del NetBeans IDE 8.0 (Public, 2015) que procesa el fichero log y extrae los datos necesarios, generando la matriz de adyacencia (ver Tabla 2) y su grafo correspondiente (se obtuvo utilizando la librería jgrapht-0.9.1 (Naveh and Contributors, 2015) desarrollada en java ver figura 3).

Tabla 2. Ejemplo de la matriz de adyacencia original.

\begin{tabular}{|c|c|c|c|c|c|c|c|c|c|}
\hline Páginas & 1 & 2 & 3 & 4 & 5 & 6 & 7 & 8 & 9 \\
\hline 1 & 1 & 1 & 1 & 0 & 0 & 1 & 1 & 0 & 1 \\
\hline 2 & 1 & 0 & 1 & 1 & 0 & 1 & 0 & 0 & 0 \\
\hline 3 & 1 & 0 & 0 & 1 & 1 & 0 & 1 & 1 & 1 \\
\hline 4 & 1 & 0 & 1 & 0 & 1 & 0 & 1 & 0 & 1 \\
\hline 5 & 1 & 1 & 0 & 1 & 0 & 1 & 1 & 0 & 1 \\
\hline 6 & 1 & 0 & 1 & 1 & 1 & 0 & 1 & 1 & 0 \\
\hline 7 & 1 & 1 & 0 & 1 & 0 & 1 & 0 & 0 & 1 \\
\hline 8 & 1 & 0 & 1 & 0 & 1 & 1 & 1 & 0 & 0 \\
\hline 9 & 1 & 1 & 1 & 1 & 1 & 1 & 0 & 1 & 0 \\
\hline
\end{tabular}

Fuente: Elaboración propia. 
Figura 3: Grafo de la matriz inicial.

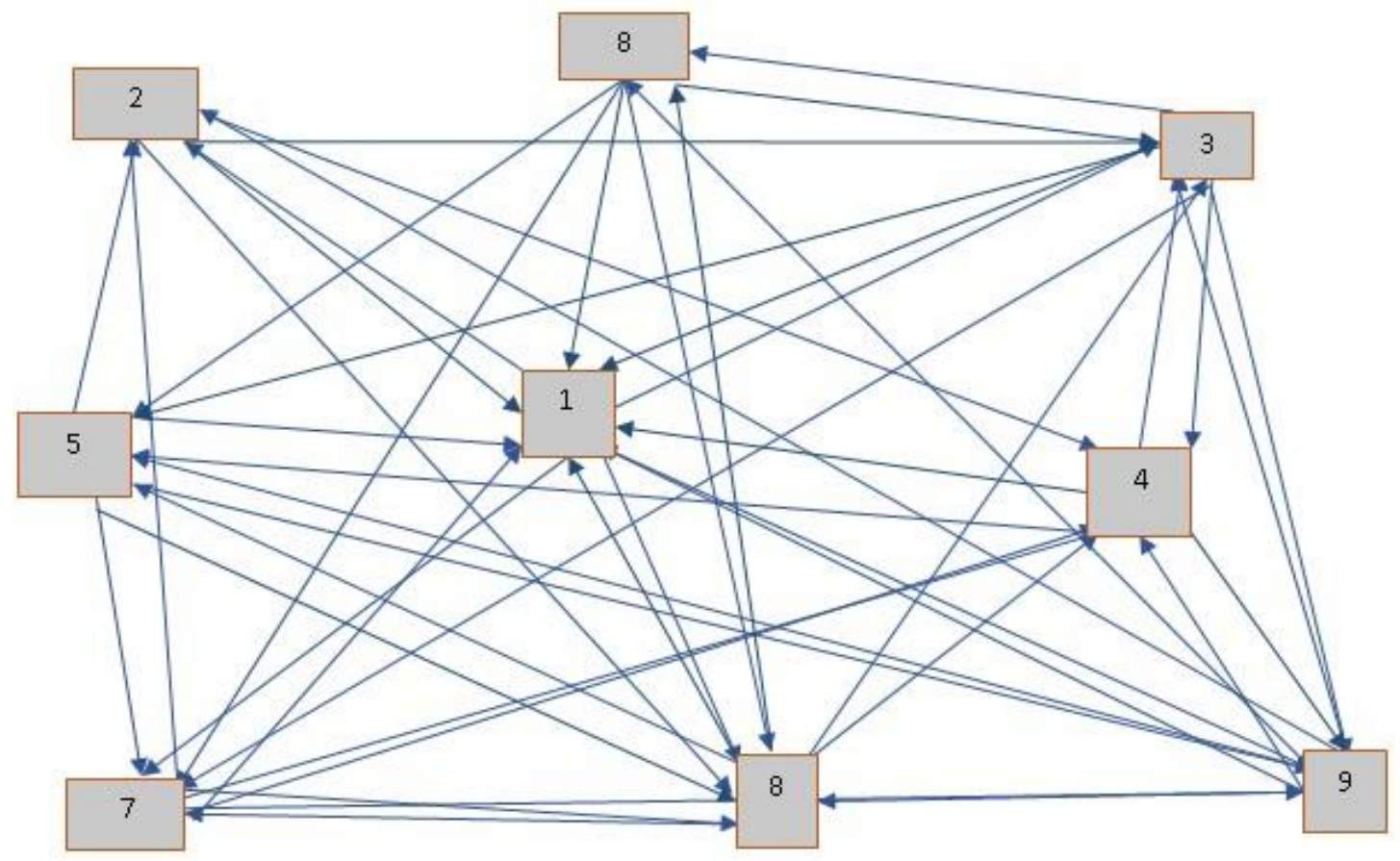

Fuente: Grafo resultante de la matriz de adyacencia inicial usando la librería jgrapht0.9.1.

Una vez obtenida la matriz de adyacencia inicial, se linealiza la matriz concatenando las filas en un solo gran vector. Después se realizan una serie de corridas de prueba a modo de análisis de sensibilidad de los parámetros a utilizar. El objetivo es determinar la combinación de parámetros que otorga un mejor resultado dado por valores numéricos en la suma final de los pesos existentes. Los parámetros seleccionados para este experimento se pueden observar en la Tabla 3.

Tabla 3: Parámetros del algoritmo AG.

\begin{tabular}{cc}
\hline Parámetro & Método \\
\hline Escala del Fitness & Rankin \\
Selección & Ruleta \\
Fracción de Cruce & 0,8 \\
Cruce & Disperso \\
Mutación & Gaussiana \\
\hline
\end{tabular}

Fuente: Elaboración propia. 
La escala del fitness se realiza mediante el método Rankin, el cual clasifica los puntajes de cada individuo basado en un ranking global de ellos. Una selección vía ruleta, simula una rueda con el área de cada segmento proporcional al fitness. La fracción de cruce por otro lado, especifica la fracción de la próxima generación que será producida vía cruce. El proceso de cruce es del tipo disperso, es decir, crea un vector binario el cual selecciona los genes respectivos del padre si su valor es 1 , y los genes de la madre si su valor es 0 , combinando los genes para formar el hijo. Finalmente, la mutación gaussiana cambia un número aleatorio a cada vector de entrada, cuyo gen afectado es tomado desde una distribución gaussiana centrada en cero.

Los otros operadores claves en la ejecución del algoritmo, tienen que ver con los criterios de detención, que son aquellos que determinan su interacción al momento de encontrar un buen ajuste dado por ciertos parámetros.

Ellos corresponden a un número máximo de generaciones, a un factor de tolerancia que indique el grado de diferencia entre el mejor individuo detectado entre las distintas generaciones y a un conteo de las mismas. El primero se fija en 10.000, el segundo se toma como nulo, y la cantidad de generaciones en que el mejor individuo detectado se mantenga (no hay otro individuo que tenga mejor fitness) se fija en 1000.

Con este último valor se está diciendo que, si el mejor individuo se mantiene como mejor individuo por sucesivas generaciones, su calidad de óptimo es cada vez mayor. Estos parámetros pueden variar y están en dependencia de cada problema, es decir estos valores varían de un problema a otro hasta encontrar los que mejor se ajustan (los que mejor resultado devuelvan).

La configuración de este modelo requiere ajustar los valores de las constantes $\varphi_{\mathrm{y}} \rho_{\text {, lo }}$ que se hace calculando sus valores mediante la Ecuación 2.6 y la Ecuación 2.7 respectivamente de la matriz inicial. Así, se busca hacer comparable ambos términos, de modo que el modelo no busque los extremos: minimizar la cantidad de enlaces o bien maximizarla de manera independiente; lo que resultaría en la matriz nula o unitaria respectivamente. Así, los cálculos en la matriz inicial resultaron ser una cantidad de enlaces iniciales de 50 y un beneficio de 1,02 . Por lo que se estudia empíricamente estos valores disminuyendo o manteniendo el valor inicial. Finalmente se fijó $\varphi_{=50 \text { y }} \rho_{=0,3}$ al proporcionar una mejor matriz resultante y adecuada convergencia.

La Tabla 4, indica los resultados del mejor individuo resultante del modelo (es el individuo que mayor valor de fitness después de 1000 generaciones (condición de parada del AG) sin mejorar este valor). La nueva adyacencia contempla 45 enlaces. El valor de la función objetivo fue incrementado y se redujo la cantidad de enlaces con respecto a la matriz inicial. 
Tabla 4: Ejemplo de la tabla de adyacencia final.

\begin{tabular}{|c|c|c|c|c|c|c|c|c|c|}
\hline Páginas & 1 & 2 & 3 & 4 & 5 & 6 & 7 & 8 & 9 \\
\hline 1 & 0 & 1 & 1 & 1 & 1 & 1 & 1 & 1 & 1 \\
\hline 2 & 1 & 0 & 1 & 0 & 0 & 1 & 1 & 1 & 0 \\
\hline 3 & 1 & 0 & 0 & 0 & 0 & 0 & 1 & 1 & 1 \\
\hline 4 & 1 & 0 & 0 & 0 & 1 & 0 & 1 & 0 & 0 \\
\hline 5 & 1 & 1 & 0 & 1 & 0 & 1 & 1 & 0 & 1 \\
\hline 6 & 1 & 0 & 0 & 1 & 1 & 0 & 1 & 0 & 0 \\
\hline 7 & 1 & 1 & 0 & 1 & 0 & 1 & 0 & 0 & 1 \\
\hline 8 & 1 & 0 & 0 & 0 & 1 & 1 & 1 & 0 & 0 \\
\hline 9 & 1 & 1 & 1 & 0 & 1 & 1 & 0 & 1 & 0 \\
\hline
\end{tabular}

Fuente: Elaboración propia.

El grafo final que representa al individuo con mayor valor de la función objetivo o fitness (mejor individuo) después de cumplirse las condiciones de parada, y, por ende, la nueva estructura web propuesta por el modelo, puede observarse en la Figura 4.

Resaltan por su importancia (valor del peso) los enlaces desde y hacia la página principal Home y los enlaces entre las páginas 2 (Árboles binarios) y 3 (Árboles Binarios de Búsqueda).

Figura 4: El grafo final que representa el mejor individuo.

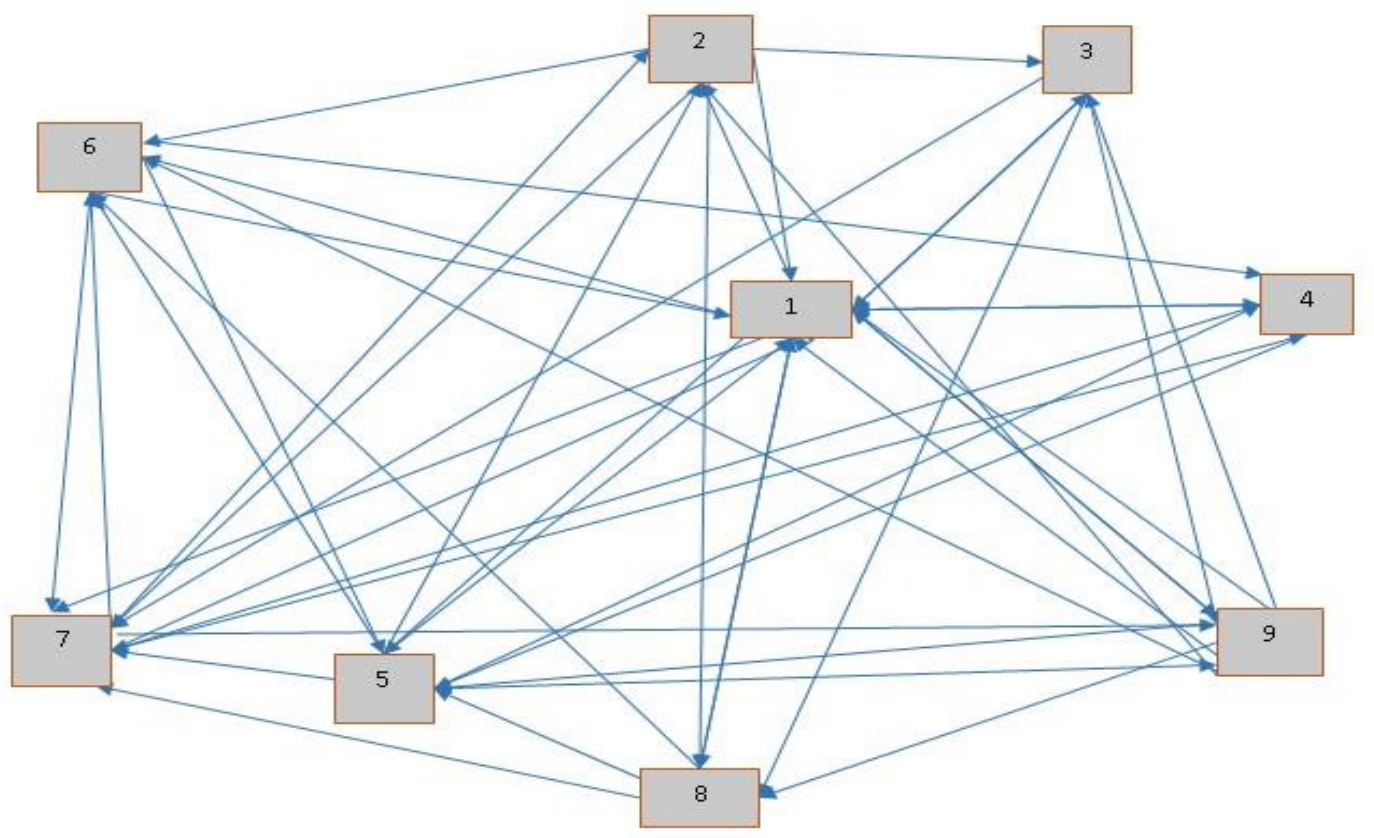

Fuente: Grafo resultante de la matriz de adyacencia final usando la librería jgrapht0.9.1. 
La nueva estructura propuesta permite la optimización de los cursos de las plataformas elearning a través de la información almacenada en los ficheros log, lo cual complementa los estudios realizados por los autores: (Zaíane and Luo, 2001; Romero et al., 2009a; Lorena, 2010; Aponte et al., 2012; Ballesteros et al., 2013 y Chakurkar and Adiga, 2015).

\section{Conclusiones.}

- Se ha realizado un estudio de la modelación matemática, particularizando en el análisis de la Minería de Uso Web con el objetivo de darle solución a las desventajas que se generan en torno del empleo por parte de los alumnos de la plataforma elearning.

- Se ha fundamentado y demostrado la importancia de la Minería Web, permitiendo extraer de los archivos log de las plataformas e-learning la información necesaria para la aplicación del modelo matemático, a partir de la matriz de adyacencia del log y la utilidad o peso de cada enlace.

- El modelo matemático propuesto permite optimizar y personalizar la estructura de los cursos en las plataformas e-learning, considerando el supuesto de que a mayor tiempo de permanencia en una página de la misma sesión, mayor interés en ella, y que la frecuencia de uso de las interrelaciones indica una mayor utilidad de las mismas para acceder hacia la información deseada, se logró encontrar estructuras de navegación más eficientes desde el punto de la navegabilidad y usabilidad, mejorando en concreto la estructura de los cursos de las plataformas e-learning en la UNAH.

\section{Referencias bibliográficas.}

Aponte, F. A., Hoyos, J. G. \& Monsalve, J. A (2012). Minería de usabilidad aplicada a plataformas virtuales de aprendizaje.

Ballesteros, A., Sánchez-Guzmán, D. \& García, R. S (2013). Minería de datos educativa: Una herramienta para la investigación de patrones de aprendizaje sobre un contexto educativo.

CLARK, R. C. \& MAYER, R. E (2011). E-learning and the science of instruction: Proven guidelines for consumers and designers of multimedia learning, John Wiley \& Sons.

CORSO, C. L. \& ALFARO, S. L. 2007. Minería de Uso Web: Presentación de caso práctico para el mejoramiento de la educación, basada en el uso de plataformas virtuales.

Chakurkar, M. \& Adiga, D (2015). A Web Mining Approach for Personalized E-Learning System. (IJACSA) International Journal of Advanced Computer Science and Applications, 5. 
HORTON, W. K (2000). Designing web-based training: how to teach anyone anything anwhere anytime. New York.

Lorena, I. C. C (2010). Minería de uso Web: Presentación de Caso práctico para el mejoramiento de la educación, basada en el uso de plataformas virtuales.

Machín, A (2016). Modelación matemática para optimizar la estructura de los cursos en las plataformas e-learning. Tesis presentada en opción al grado científico de Máster en Biomatemática. Universidad Agraria de la Habana. Mayabeque. Cuba. 60p.

Menascé, D. A., Almeida, V. A., Fonseca, R. \& Mendes, M. A (1999). A methodology for workload characterization of e-commerce sites. Proceedings of the 1st ACM conference on Electronic commerce. ACM, 119-128.

Naveh, B. \& Contributors (2015). JGraphT [Online]. Available: http://jgrapht.org/ [Accessed 5 de octubre 2020].

Ortega-Priego, J.-L. \& Aguillo, I. F (2009). Minería del uso de webs. El profesional de la información, 18, 20-26.

Public, G. G (2015). Netbeans IDE 8.0 [Online]. [Accessed 6 de septiembre 2020].

Romero, M. C., Ventura, S. S. \& Hervás, M. C (2009b). Estado actual de la aplicación de la minería de datos a los sistemas de enseñanza basada en web.

Rosenberg, M. J. (2001). E-learning: Strategies for delivering knowledge in the digital age, McGraw-Hill New York.

Rosenberg, M. J (2005). The Future of e-Learning. ASTD's Learning Circuits Webzine.

SILVIO, J (2004). Tendencias de la educación superior virtual en América Latina y el Caribe. La educación superior virtual en América Latina y el Caribe, 15-39.

Vázquez, Y (2008). Sistema de Objetos de Aprendizaje para la enseñanza de la Matemática Básica en la carrera Ingeniería Agronómica. Tesis en opción al Título Académico de Master en Nuevas Tecnologías para la Educación. Universidad Agraria de la Habana. La Habana. Cuba.84p.

Velásquez, J. D. \& Palade, V (2008). Adaptive web sites: A knowledge extraction from web data approach. Proceedings of the 2008 conference on Adaptive Web Sites: A Knowledge Extraction from Web Data Approach. IOS Press, 1-272.

Zaíane, O. R. \& Luo, J (2001). Web usage mining for a better web-based learning environment. Proceedings of conference on advanced technology for education. 6064.

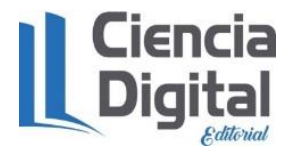




\section{Para citar el artículo indexado}

Machín Armenteros, A., \& Vázquez Alfonso, Y. (2020). Modelación matemática para optimizar la estructura de los cursos en las plataformas e-learning. AlfaPublicaciones, 1(1). https://doi.org/10.33262/ap.v1i1.17

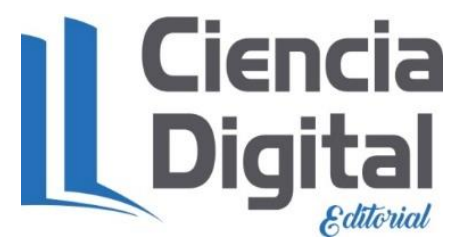

El artículo que se publica es de exclusiva responsabilidad de los autores y no necesariamente reflejan el pensamiento de la Revista Alpha Publicaciones.

El artículo queda en propiedad de la revista y, por tanto, su publicación parcial y/o total en otro medio tiene que ser autorizado por el director de la Revista Alpha Publicaciones.
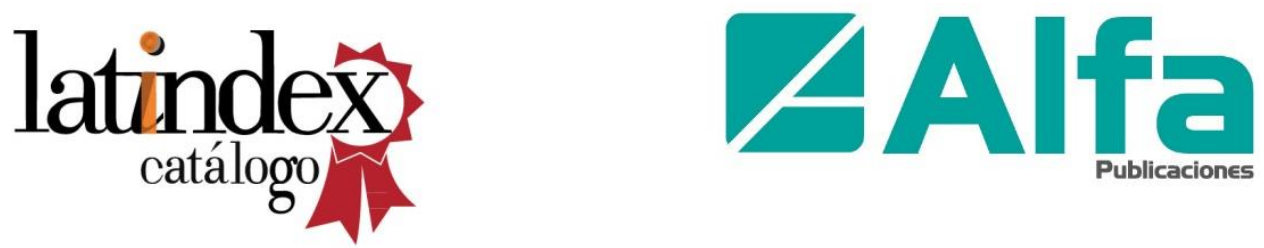